\title{
MODELLING HEAT AND MASS TRANSFER IN HIGH-PRESSURE FOOD PROCESSING
}

\author{
Otero, L. ${ }^{\mathrm{a}}$; Ramos, A. M. ${ }^{\mathrm{b}}$; de Elvira, C. ${ }^{\mathrm{a}}$ and Sanz, P. D. ${ }^{\mathrm{a} *}$ \\ ${ }^{a}$ Department of Engineering. Instituto del Frío. CSIC. \\ c/ José Antonio Novais, 10. 28040 Madrid. Spain. \\ ${ }^{\mathrm{b}}$ Department of Applied Mathematics. Facultad de Matemáticas. Universidad Complutense \\ de Madrid. Ciudad Universitaria, s/n. 28040 Madrid. Spain.
}

\begin{abstract}
.
The objective of this study is to obtain a mathematical model to describe the phenomena of heat and mass transfer taking place during pressurization of a food model. High-pressure experiments were carried out in agar gel samples. During all the experiments, the temperature evolution in the centre and in the surface of the samples were recorded. The mathematical model took into account free convection arising in the pressure medium after compression as it is known its influence in the thermal evolution of the processed food.-Performed simulations performed showed good agreement between experimental and predicted temperature profiles for all the studied processes. Modelling of high-pressure processes is important for their optimization and their general implantation in the food industry.
\end{abstract}

Keywords: modelling, high-pressure, processing.

\section{Text.}

High-pressure processing is an expanding technology that is being successfully introduced in the food industry mainly as a preservation method to control the microbiological and enzymatic activities in food. In the last years, an increasing interest has been drawn in temperature uniformity of high-pressure processes (Farkas \& Hoover, 2000; Ting, Balasubramaniam, \& Raghubeer, 2002). Process inhomogeneities may lead to insufficient inactivation of microorganisms and enzymes being very likely the reason of different inactivation results found in the literature. Moreover, it must be taken into account that most studies are performed using laboratory-scale equipment and temperature histories may become quite different when the same treatment is carried out in a large-scale equipment. The aim of this paper is to obtain a mathematical model to describe the phenomena of heat and mass transfer taking place during the high-pressure treatment of solid foods. The model will allow to evaluate the influence of free convection arising in the pressure medium after compression over the thermal evolution of the processed solid food. The final objective is to employ the model as a tool to improve the temperature control in the sample during the pressure treatment with the aim of achieving a high degree of process uniformity (Otero et al., 2006).

High-pressure experiments were carried out in a pilot unit (ACB GEC Alsthom, Nantes, France) which was previously filled with water, acting as pressure medium, and tempered to $20 \pm 0.5^{\circ} \mathrm{C}$ during at least 24 hours before the experiments by a lateral surrounding isolated coil connected to a thermoregulating system. Dimensions of the cylindrical pressure vessel were $0.1 \mathrm{~m}$ diameter and $0.3 \mathrm{~m}$ height. High-pressure treatments were performed in agar samples of two different sizes which implied different filling ratios in the pressure vessel: for big samples filling ratio was about $71 \%$ while it was around $12 \%$ for small ones. This allowed to show the relative influence of the free convection currents aris-

*E-mail: psanz@if.csic.es 
ing in the pressure medium after compression over the solid sample thermal evolution. Coupled heat and mass equations defining the problem in the considered domain were solved using the finite element method with the aid of a commercial software FEMLAB ${ }^{\circledR}$, Release 3.1i, and the corresponding Heat Transfer Module 3.1 (COMSOL AB, Sweden).

Figure 1 clearly shows the importance of free convection when the filling ratio in the pressure vessel is low (Figure 1b). In this case, in order to accurately describe the temperature evolution in the solid sample, both heat and mass transport in the pressure medium have to be taken into account when modelling. Figure 1 also shows the non-homogeneous temperature throughout the sample during its processing. Our model allowed the design of processes in which temperature is maintained constant throughout the product, independently of
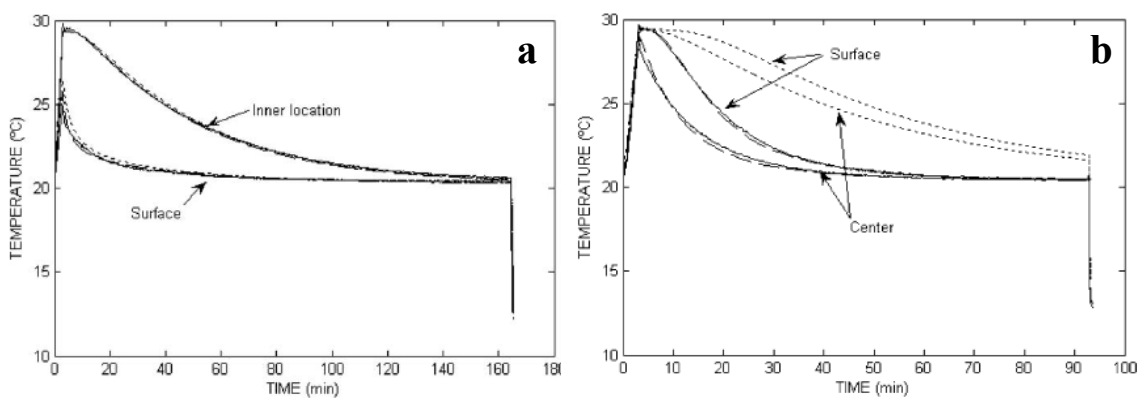

Figure 1.-Temperature evolution in (a) big and (b) small agar samples during a pressure treatment at $350 \mathrm{MPa}$. (-): Experimental data; $(\cdots)$ : Predicted values when only conduction is considered; (--): Predicted values when conduction and convection are considered as heat transfer mechanisms in the pressure medium.
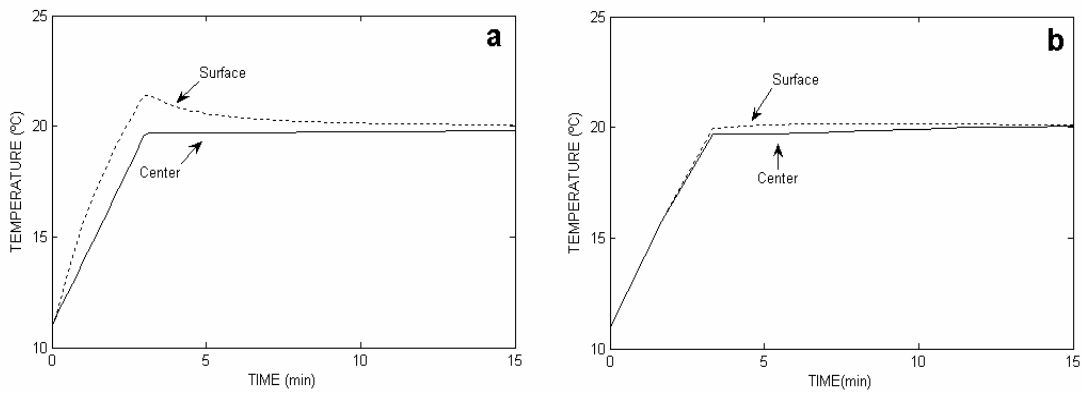

Figure 2.- Temperature evolution calculated from the model in (a) big and (b) small agar samples during a pressure treatment at $350 \mathrm{MPa}$.

product processed per cycle, assuring a high degree of uniformity. its size (Figure 2).

Therefore, the mathematical model described in this paper provides a useful tool to simulate the heat and mass transfer phenomena that occur during the pressure processing of food and to design processes seeking an uniform temperature distribution taking into account the filling ratio. For real processes to be effective and profitable, it is necessary to reach an equilibrium between the needed thermal control in the sample and the amount of

\section{Acknowledgements.}

This research was carried out with financial support from the AGL 2003-06862-C02-00 M.E.C Spain project. L. Otero was supported by a Ramón y Cajal R. C. from M.E.C. Spain.

\section{Literature cited.}

Farkas, D. F., \& Hoover, D. G. 2000. High pressure processing. Journal of Food Science Supplement: $\quad$ Kinetics of Microbial Inactivation for Alternative Food Processing Technologies, 47-64.

Otero, L.; Ramos, A. M.; de Elvira, C. and Sanz, P. D. 2006. A model to design high-pressure processes towards an uniform temperature distribution. Journal of Food Engineering (in press).

Ting, E., Balasubramaniam, V. M., \& Raghubeer, E. 2002. Determining thermal effects in highpressure processing. Food Technology, 56 (2), 31-35. 